\title{
The E1784K mutation in SCN5A is associated with mixed clinical phenotype of type 3 long QT syndrome
}

\author{
Naomasa Makita, ${ }^{1}$ Elijah Behr, ${ }^{2}$ Wataru Shimizu, ${ }^{3}$ Minoru Horie, ${ }^{4}$ Akihiko Sunami, ${ }^{5}$ \\ Lia Crotti, ${ }^{6}$ Eric Schulze-Bahr, ${ }^{7}$ Shigetomo Fukuhara, ${ }^{8}$ Naoki Mochizuki, ${ }^{8}$ Takeru Makiyama, ${ }^{9}$ \\ Hideki Itoh, ${ }^{4}$ Michael Christiansen, ${ }^{10}$ Pascal McKeown, ${ }^{11}$ Koji Miyamoto, ${ }^{3}$ Shiro Kamakura, ${ }^{3}$ \\ Hiroyuki Tsutsui, ${ }^{1}$ Peter J. Schwartz, 6,12,13 Alfred L. George Jr., ${ }^{14}$ and Dan M. Roden ${ }^{14}$
}

${ }^{1}$ Department of Cardiovascular Medicine, Hokkaido University Graduate School of Medicine, Sapporo, Japan. ${ }^{2}$ Cardiology and Cardiological Sciences, St. George's University of London, London, United Kingdom. ${ }^{3}$ Division of Cardiology, National Cardiovascular Center, Suita, Japan. ${ }^{4}$ Department of Cardiovascular Medicine, Shiga University of Medical Science, Otsu, Japan. ${ }^{5}$ Department of Pharmaceutical Sciences, International University of Health and Welfare, Ohtawara, Japan. ${ }^{6}$ Section of Cardiology, Department of Lung, Blood and Heart, University of Pavia, and Department of Cardiology and Molecular Cardiology Laboratory, IRCCS Fondazione Policlinico S. Matteo, Pavia, Italy. 'Department of Cardiology and Angiology, University Hospital of Muenster, and Leibniz Institute for Arteriosclerosis Research at the University of Muenster, Muenster, Germany. ${ }^{8}$ Department of Structural Analysis, National Cardiovascular Center Research Institute, Suita, Japan. ${ }^{9}$ Department of Cardiovascular Medicine, Kyoto University Graduate School of Medicine, Kyoto, Japan. ${ }^{10 D e p a r t m e n t ~ o f ~ C l i n i c a l ~ B i o c h e m i s t r y, ~ S t a t e n s ~ S e r u m ~ I n s t i t u t, ~ C o p e n h a g e n, ~ D e n m a r k . ~}$ ${ }^{11}$ Institute of Clinical Science, Queen's University Belfast and Royal Victoria Hospital, Belfast, United Kingdom. ${ }^{12}$ Laboratory of Cardiovascular Genetics, IRCCS Istituto Auxologico, Milan, Italy. ${ }^{13}$ Cardiovascular Genetics Laboratory, Hatter Institute for Cardiovascular Research, Department of Medicine, University of Cape Town, Cape Town, South Africa. ${ }^{14}$ Department of Pharmacology and Department of Medicine, Vanderbilt University, Nashville, Tennessee, USA.

\begin{abstract}
Phenotypic overlap of type 3 long QT syndrome (LQT3) with Brugada syndrome (BrS) is observed in some carriers of mutations in the Na channel SCN5A. While this overlap is important for patient management, the clinical features, prevalence, and mechanisms underlying such overlap have not been fully elucidated. To investigate the basis for this overlap, we genotyped a cohort of 44 LQT3 families of multiple ethnicities from 7 referral centers and found a high prevalence of the E1784K mutation in SCN5A. Of $41 \mathrm{E} 1784 \mathrm{~K}$ carriers, $93 \%$ had LQT3, 22\% had BrS, and 39\% had sinus node dysfunction. Heterologously expressed E1784K channels showed a 15.0-mV negative shift in the voltage dependence of $\mathrm{Na}$ channel inactivation and a 7.5-fold increase in flecainide affinity for resting-state channels, properties also seen with other LQT3 mutations associated with a mixed clinical phenotype. Furthermore, these properties were absent in Na channels harboring the T1304M mutation, which is associated with LQT3 without a mixed clinical phenotype. These results suggest that a negative shift of steady-state $\mathrm{Na}$ channel inactivation and enhanced tonic block by class IC drugs represent common biophysical mechanisms underlying the phenotypic overlap of LQT3 and BrS and further indicate that class IC drugs should be avoided in patients with $\mathrm{Na}$ channels displaying these behaviors.
\end{abstract}

\section{Introduction}

Congenital long QT syndrome (LQTS) is characterized by the prolongation of the QT interval on surface ECGs and an increased risk of potentially fatal ventricular arrhythmias, especially torsade de pointes (1). QT interval is determined by the cardiac action potential which is orchestrated by a fine balance between inward and outward currents expressed in myocardial cells. Mutations in $S C N 5 A$, the gene encoding the most prevalent cardiac $\mathrm{Na}$ channel $\alpha$ subunit, are responsible for a spectrum of hereditary arrhythmias including type 3 LQTS (LQT3) (2), Brugada syndrome (BrS) (3), cardiac conduction disturbances (4), and sick sinus syndrome (SSS) (5). More than 70 distinct SCN5A mutations responsible for LQT3 have been reported (see http://www.fsm.it/cardmoc), and the common in vitro consequence of most of these mutations is a persistent

Nonstandard abbreviations used: $A_{\mathrm{f}}$, fraction of the fast inactivating component; $A_{\mathrm{s}}$, fraction of the slow inactivating component; BrS, Brugada syndrome; LQT3, type 3 LQTS; LQTS, long QT syndrome; QTc, rate-corrected QT interval calculated using Bazett's formula; SSS, sick sinus syndrome; $\tau_{f}$, time constant of the fast inactivating component; $\tau_{\mathrm{s}}$, time constant of the slow inactivating component; TTX, tetrodotoxin; UDB, use-dependent block; $V_{1 / 2}$, half-maximal voltage.

Conflict of interest: The authors have declared that no conflict of interest exists. Citation for this article: J. Clin. Invest. 118:2219-2229 (2008). doi:10.1172/JCI34057.
$\mathrm{Na}$ current during the action potential plateau due to destabilized fast $\mathrm{Na}$ channel inactivation (2). This failure of fast inactivation shifts the ionic balance during the plateau phase toward inward current and delays repolarization, thus increasing action potential duration and the corresponding QT interval. Na channel blockers such as mexiletine (class IB) or flecainide (class IC) shorten QT in LQT3 due to block of this persistent current (6-8) and have therefore been used in the management of affected patients.

Despite these common biophysical features, the clinical manifestations associated with LQT3 mutations are variable. Surprisingly, some LQT3 patients display ECG findings characteristic of $\mathrm{BrS}$ (ST elevation in the right precordial leads) that are related to another biophysical mechanism, a reduction of peak $\mathrm{Na}$ current (9). Reduced Na current is thought to exaggerate differences in action potential duration between the inner (endocardium) and outer (epicardium) layers of ventricular muscle, thereby favoring a substrate promoting reentrant arrhythmias. Phenotypic overlap between LQT3 and BrS was first reported in a large Dutch family with an insertion mutation $1795 \mathrm{ins}$, in which the mutation carriers showed ECG features of both LQT3 and BrS $(10,11)$. Importantly, $\mathrm{Na}$ channel block in the overlap phenotype shortens QT but exacerbates the ST segment elevation BrS phenotype 

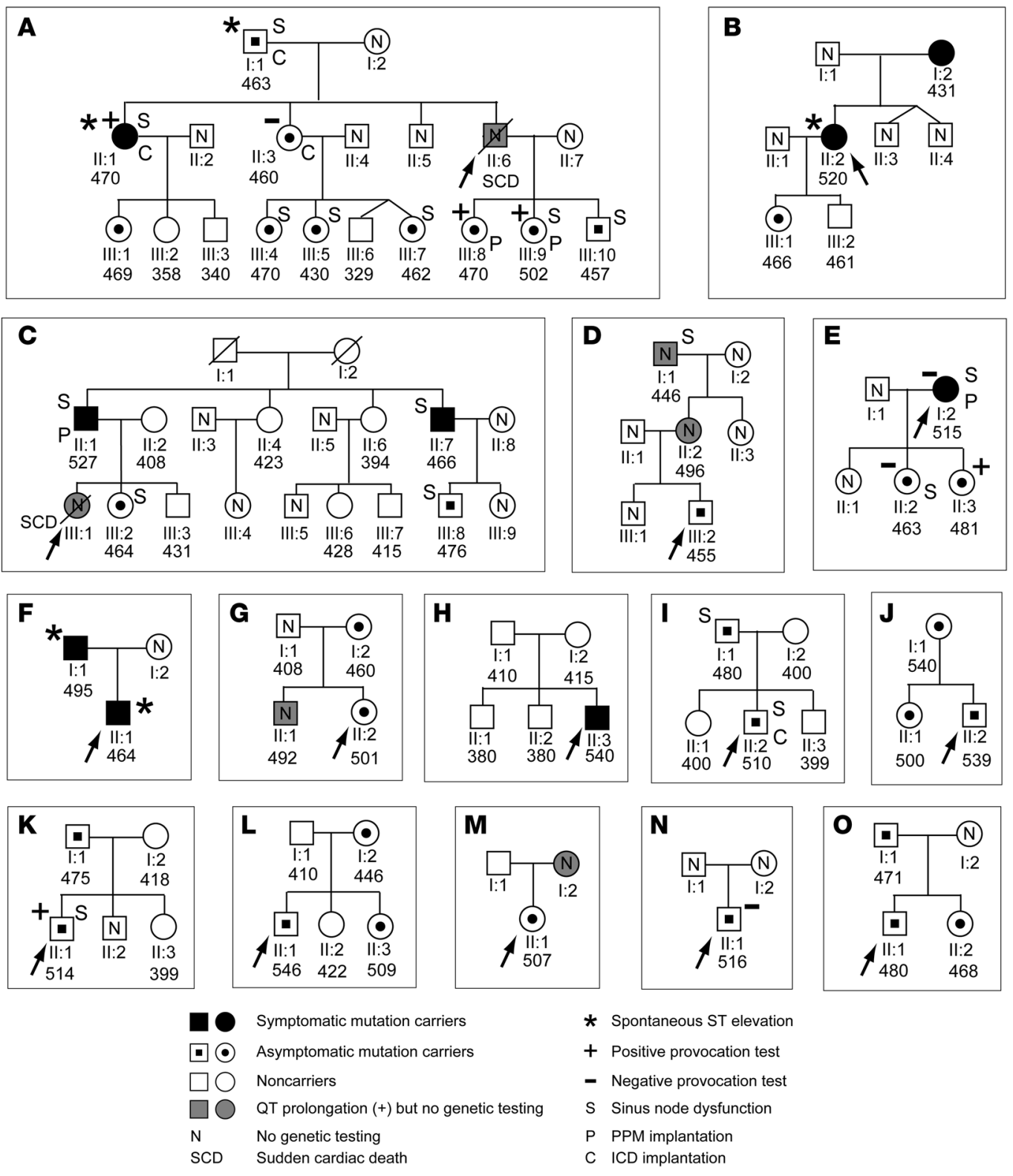

$$
\begin{aligned}
& \text { * Spontaneous ST elevation } \\
& \text { + Positive provocation test } \\
& \text { - Negative provocation test } \\
& \text { S Sinus node dysfunction } \\
& \text { P PPM implantation } \\
& \text { C ICD implantation }
\end{aligned}
$$

Figure 1

Pedigrees of E1784K families. Pedigrees of 15 LQT3 families (pedigrees A-O shown in panels A-O, respectively) carrying E1784K are shown. The pedigree $C$ was previously reported elsewhere (17). Probands are indicated by an arrow. Nine symptomatic mutation carriers, shown by the filled symbols, had episodes of syncope $(n=8)$ and unexplained palpitations $(n=1$; B;Il:2). Asymptomatic mutation carriers $(n=32)$ are shown as symbols with a dot, and gray symbols are the individuals with QT prolongation who declined genetic testing, or are sudden cardiac death victims (SCD; A;II:6 and C;III:1) whose DNA was not available. Individuals exhibiting ST elevation in the right precordial leads are depicted with an asterisk. N represents individuals genetically undetermined. S, P, and C represent individuals who had sinus node dysfunction $(n=16)$, permanent pacemaker implantation (PPM; $n=4)$, and an implantable cardioverter defibrillator (ICD; $n=4)$, respectively. Values for QTc intervals are given beneath each symbol. The Na channel provocation test was positive in individuals with + (A;II:1, A;III:8, A;III:9, E;II:3, and $K ; \| l 1)$, and negative in the individuals with - (A;II:3, E;I:2, E;II:2, and N;II:1). In family G, V1098L was identified in II:2 (who also had E1784K) but not in I:2 (an E1784K carrier), showing that II:2 was a compound SCN5A mutation carrier. In family H, parents of the proband were both genetically unaffected, indicating that E1784K in the proband was most likely a de novo mutation. Three asymptomatic family members with a prolonged QTc (D;I:1, D;Il:2, and G;Il:1) declined genetic testing, and 1 individual, D;l:1, exhibited sinus node dysfunction. Squares and circles indicate men and women, respectively.

and thus enhances arrhythmia risk (11). Biophysical studies demonstrated that the mutant channels displayed enhancement of both closed-state inactivation and slow inactivation, and this was thought to sensitize carriers to the BrS phenotype during flecainide therapy (12).
The overlap between the LQT3 and BrS phenotypes has also been reported in other SCN5A mutations such as $\triangle \mathrm{KPQ}(13,14)$, E1784K (13), and $\Delta K 1500(15)$, raising a concern about the safety of class IC drug therapy in LQT3 patients (13) and questions about the underlying mechanisms. However, phenotypic variability in LQT3 
A

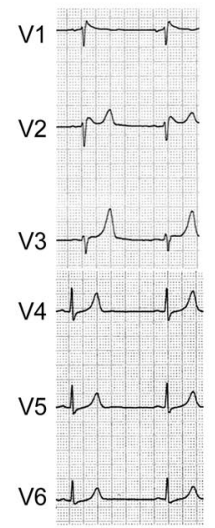

B
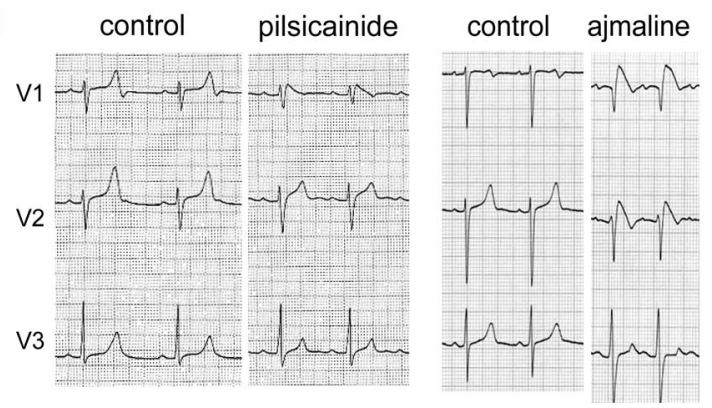

D

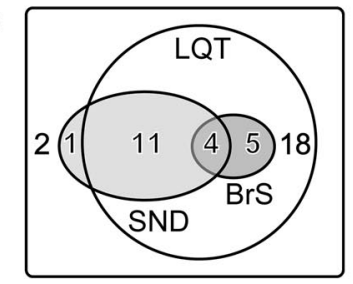

\section{Figure 2}

ECG characteristics of E1784K mutation carriers. (A) QT prolongation (QTc, $470 \mathrm{~ms}$ ) and spontaneous saddleback type ST elevation observed in the right precordial leads in carrier A;Il:1. (B) ECG recordings before and after the $\mathrm{Na}$ channel blocker provocation test. Pilsicainide (left, patient K;II:1) induced coved-type ST elevation in V1 and the QTc was concomitantly shortened (QTc: control, $495 \mathrm{~ms}$; pilsicainide, $459 \mathrm{~ms}$ ). Ajmaline (right, patient A;III:9) also induced coved-type ST elevation in V1 and V2 and QTc shortening (control, $501 \mathrm{~ms}$; ajmaline, $490 \mathrm{~ms}$ ). (C) Sinus node dysfunction (SND) demonstrated by a 3.9-s sinus arrest in carrier A;l:1. (D) A Venn diagram representing electrophysiological manifestation of 41 SCN5A-E1784K mutation carriers. Thirtyeight carriers exhibited an abnormally long QTc, 3 individuals had a normal QTc, and 1 exhibited sinus node dysfunction only. Sinus node dysfunction and BrS were observed in 16 and 9 individuals, respectively, with 4 displaying both phenotypes. has thus far been reported sporadically or only within a single kindred. Therefore, it is not clear whether development of the BrS phenotype in a patient with LQT3 is solely determined by the biophysical properties of the mutant channel or by coinherited genetic variations, gender, ethnicity, or other environmental factors. One approach to dissect such phenotypic variability is to perform a clinical assessment of individuals with multiple pedigrees from genetically heterogeneous populations with the same mutation.

In the present study, we evaluated 15 kindreds of diverse ethnic backgrounds from Asia, Europe, and North America, all with the same LQT3 mutation, E1784K. We report here a high prevalence of overlap of the LQT3 phenotype with BrS and sinus node dysfunction. Furthermore, we have identified biophysical and pharmacological properties of the mutant channels that appear to be common to other mutants with this clinical overlap, thereby suggesting certain features of the mutant $\mathrm{Na}$ channel that result in an abnormal response to class IC drugs. These data extend the concept that molecular characterization of the consequences of individual DNA variants is desirable before the selection of a therapeutic approach in LQT3 patients.

\section{Results}

Clinical phenotypes in 15 LQT3 families with SCN5A-E1784K. Among the 66 family members who underwent genetic testing, 41 were identified as heterozygous mutation carriers (18 men, 23 women, $25 \pm 19$ years, means $\pm \mathrm{SD}$ ) and 25 as noncarriers (11 men, 14 women). Three individuals showed a prolonged rate-corrected QT interval (QTc; calculated using Bazett's formula, QTc $=$ QT $/$ [previous RR interval] $]^{1 / 2}$ ) but declined genetic testing, and 2 victims of sudden cardiac death, whose DNA was not available, were presumably affected (shown with shaded symbols in Figure 1). The proband of the family G (II:2) carried the SCN5A mutation V1098L on the opposite allele, but otherwise no compound mutations (SCN5A or other LQTS genes) were observed. Among 41 carriers, 9 had episodes of syncope $(n=8)$ or unexplained palpitations $(n=1)$, but the remaining 32 individuals (78\%) were asymptomatic. The QTc interval (mean $\pm \mathrm{SD}$ ) measured from the resting 12-lead ECG of all mutation carriers (living probands and family members) was $485 \pm 30 \mathrm{~ms}$, which was significantly longer $(P<0.001)$ than the
$402 \pm 31 \mathrm{~ms}$ measured in the noncarriers. There was no significant difference in the QTc interval between male (493 $\pm 31 \mathrm{~ms}, n=18$ ) and female carriers $(479 \pm 28 \mathrm{~ms}, n=23)$. The QTc penetrance, defined as the percentage of mutation carriers with abnormally long QTc interval at presentation (>440 ms for men and >460 ms for women), was $93 \%$ (18 men, 20 women), indicating a highly penetrant LQT3 mutation. Administration of mexiletine shortened the QTc in 10/10 individuals tested, as previously demonstrated in other LQT3 mutation carriers (6). Mexiletine did not unmask or exacerbate Brugada-type ST elevation.

Spontaneous ST elevation in the right precordial leads was observed in 5/41 mutation carriers (Figure 1; coved-type, $n=1$; saddleback type, $n=4$; Figure $2 \mathrm{~A}$ ). Nine mutation carriers without diagnostic ST elevation at baseline underwent provocation with flecainide, ajmaline, or pilsicainide, and the test was positive (covedtype ST elevation; Figure 2B) in 5 (Figure 1). The 4 mutation carriers with a negative provocation test (A;II:3, E;I:2, E;II:2, and N;II:1) were not rechallenged. Thus, the diagnosis of $\mathrm{BrS}$ was established in 9/41 mutation carriers ( 1 individual, A;II:1, showed spontaneous saddleback ST elevation, which was converted to coved-type by ajmaline). None of the noncarriers displayed a Brugada-type ECG.

Sinus bradycardia and atrial standstill have also been associated with other SCN5A mutations (7, 11, 15-18), and sinus bradycardia was first reported in 1980 in LQTS patients who were subsequently found to be carriers of $\triangle \mathrm{KPQ}$ (19). Sinus node dysfunction was common in our cohort, presenting in 16/41 mutation carriers (Figure 2C), and 4 of these 16 carriers with sinus node dysfunction also exhibited the BrS phenotype (Figure 2, B and D). Moreover, one carrier (A;III:5) showed sinus node dysfunction without manifesting QT prolongation or ST elevation. Four patients received a permanent pacemaker, and another 4 received an implantable cardioverter defibrillator.

Biophysical properties and membrane trafficking of E1784K. Figure $3 \mathrm{~A}$ illustrates representative whole-cell current traces from cells expressing WT or E1784K Na channels in the presence of coexpressed human $\beta_{1}$ subunit. Peak current density of the E1784K channel was approximately $40 \%$ less than the that of the WT channel (WT, $138.2 \pm 13.0 \mathrm{pA} / \mathrm{pF}, n=25 ; \mathrm{E} 1784 \mathrm{~K}, 82.3 \pm 8.2 \mathrm{pA} / \mathrm{pF}, n=13$; $P<0.005)$ (Figure 3B). Cell capacitance of WT and E1784K was 
A
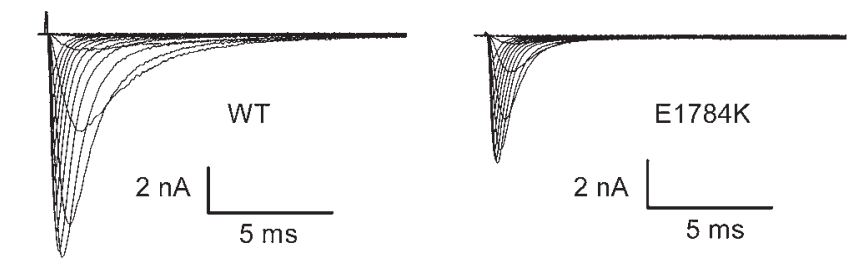

C

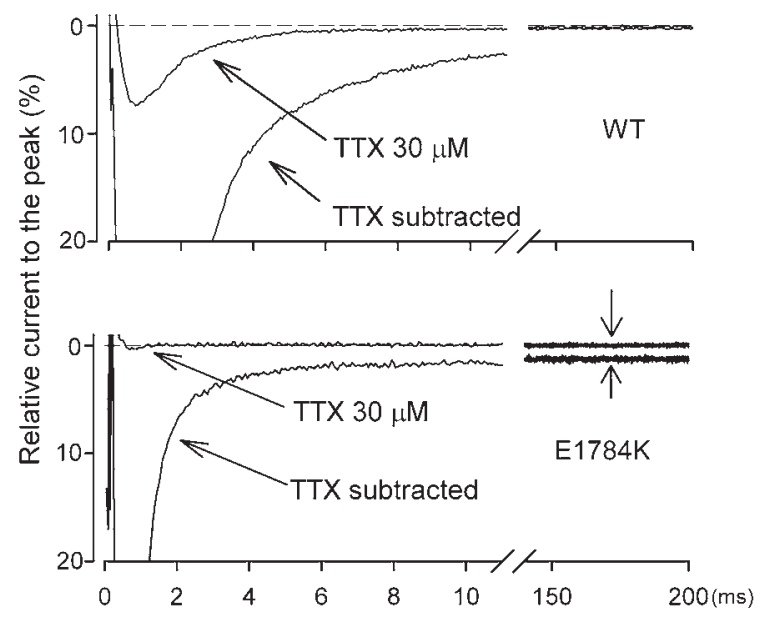

B

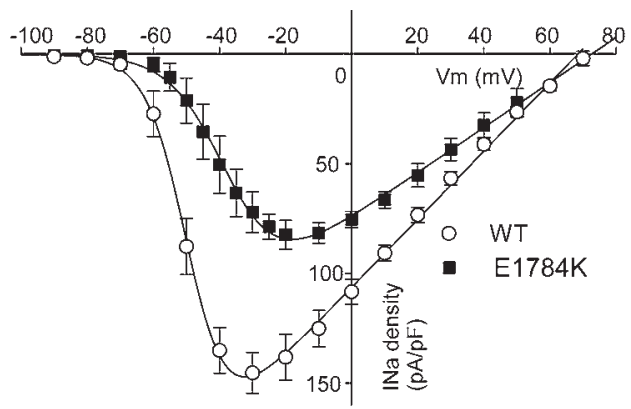

D
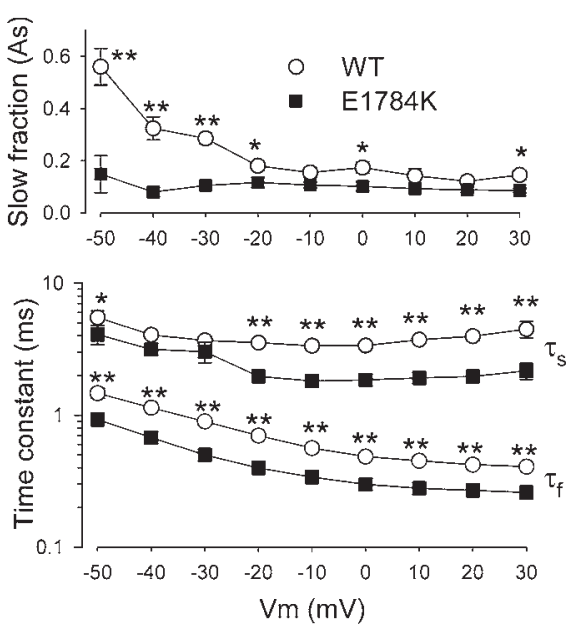

Figure 3

Properties of E1784K whole-cell current. (A) Representative whole-cell current traces obtained from tsA-201 cells transfected with either WT or E1784K Na channels. All studies were conducted in cells cotransfected with human $\mathrm{Na}$ channel $\beta_{1}$ subunits. Currents were recorded from a holding potential of $-120 \mathrm{mV}$ and stepped from $-90 \mathrm{mV}$ to $90 \mathrm{mV}$ for $20 \mathrm{~ms}$ in 10-mV increments. (B) Current-voltage relationship. Current was normalized to cell capacitance to give a measure of Na current density. There were significant differences $(P<0.05)$ in current density between WT and E1784K at all tested voltages except $-80,-70,-60$, and $\geq 40 \mathrm{mV}$. (C) Na currents were recorded with a test pulse potential of $-20 \mathrm{mV}$ from a holding potential of $-120 \mathrm{mV}$ before and after $30 \mu \mathrm{mol} / \mathrm{ITTX}$, and the TTX-sensitive current was calculated by digital subtraction. The currents were normalized to the peak current without TTX and superimposed. Zero current levels are shown by dotted lines. Note the faster decay and the prominent TTX-sensitive persistent current (shown with arrows) in E1784K. The amplitude of the persistent current was approximately 6 -fold larger in E1784K (1.85\% $\pm 0.27 \%$ of peak, $n=10 ; P<0.001)$ than in WT $(0.32 \% \pm 0.05 \%$ of peak, $n=15)$. The density of persistent current for E1784K (1.53 $\pm 0.22 \mathrm{pA} / \mathrm{pF})$ was significantly larger than for WT $(0.44 \pm 0.07 \mathrm{pA} / \mathrm{pF})$. (D) Time constants for the voltage dependence of inactivation. $A_{s}$ is shown in the upper panel, and $\tau_{f}$ and $\tau_{s}$ are presented in the lower panel. Significant differences between WT (open circles, $n=13$ ) and E1784K (filled squares, $n=13$ ) are indicated $\left({ }^{\star} P<0.05\right.$, ${ }^{\star *} P<0.01$ ).

comparable. In addition, peak Na current occurred at more positive potentials $(+14 \mathrm{mV})$ with the mutant channel than with the WT channel. However, the E1784K channel showed an increased level of tetrodotoxin-sensitive (TTX-sensitive) persistent current, a hallmark biophysical abnormality of LQT3 (Figure 3C). The density of the persistent current of E1784K after $200 \mathrm{~ms}$ of depolarization at $-20 \mathrm{mV}$ was 3.5 -fold larger than that of WT (WT, $0.44 \pm 0.07 \mathrm{pA} / \mathrm{pF}$, $n=15$; E1784K, $1.53 \pm 0.22 \mathrm{pA} / \mathrm{pF}, n=10 ; P<0.001)$ and was abolished by $30 \mu \mathrm{mol} / 1$ TTX (Figure 3C). Furthermore, macroscopic current decay was faster for E1784K than for WT. Double exponential curve fitting (see Methods) revealed that the fraction of the slow inactivating component $\left(A_{\mathrm{s}}\right)$ was significantly smaller and the time constants of both the fast and slow inactivating components $\left(\tau_{\mathrm{f}}, \tau_{\mathrm{s}}\right)$ were significantly smaller in E1784K compared with WT at most test-pulse voltages between $-50 \mathrm{mV}$ and $30 \mathrm{mV}$ (Figure $3 \mathrm{D})$. These results demonstrate that the $\mathrm{E} 1784 \mathrm{~K}$ channels display an enhanced onset of fast inactivation (faster current decay), as well as destabilized fast inactivation (increased persistent current), probably due to the fluctuation of the channel between normal and non-inactivating gating modes (2). These results are responsible for the QT prolongation phenotype.

The voltage dependence of steady-state inactivation for E1784K was abnormal because of a large $(-15.0 \mathrm{mV})$ hyperpolarizing shift (half-maximal voltage $\left[V_{1 / 2}\right]$ : WT, $-86.8 \pm 1.1 \mathrm{mV}$, $n=25 ; \mathrm{E} 1784 \mathrm{~K},-101.8 \pm 1.3 \mathrm{mV}, n=17 ; P<0.001$ ) (Figure 4A). The steady-state inactivation curves (left curves of Figure $4 \mathrm{~A}$ ) predicted decreased channel availability, a hallmark of BrS/SSS. For example, at a prepulse potential of $-90 \mathrm{mV}$, which is close to the resting membrane potential of ventricular myocytes, $58.6 \% \pm 3.5 \%$ of WT channels were available, but this was significantly attenuated in E1784K channels $(17.8 \% \pm 3.1 \%)$. Another important potential contributor to the BrS/SSS phenotype was a significant shift in the voltage dependence of activation of $\mathrm{E} 1784 \mathrm{~K}$ by $+12.5 \mathrm{mV}\left(V_{1 / 2}\right.$ : WT, $-49.7 \pm 1.1 \mathrm{mV}, n=32$; E1784K, 
A

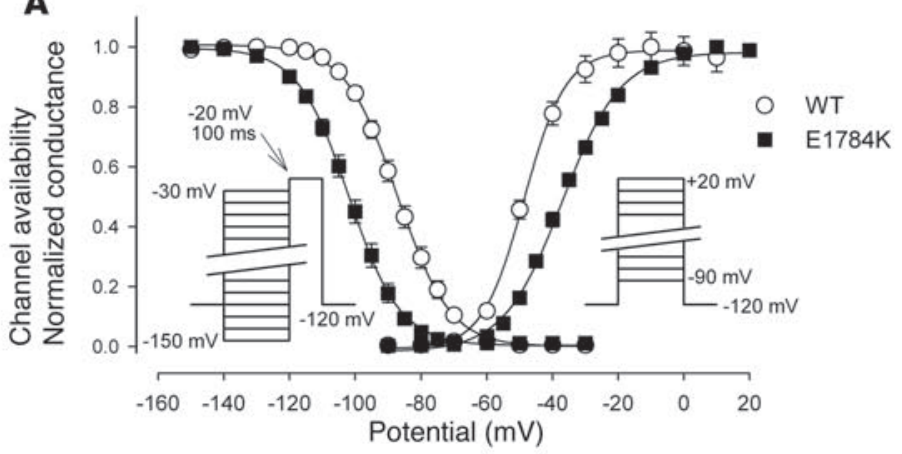

B

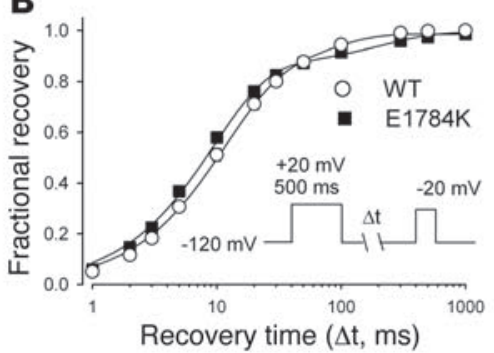

D

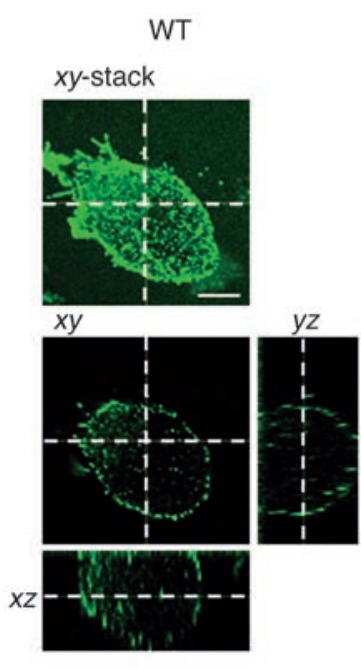

c

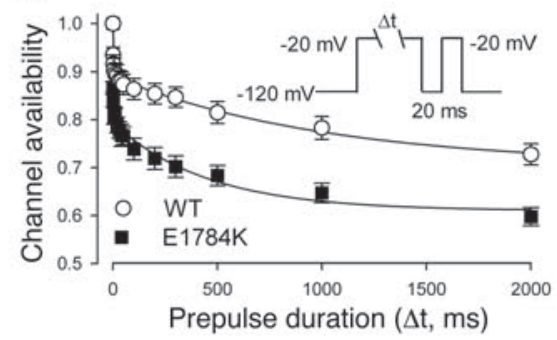

E1784K
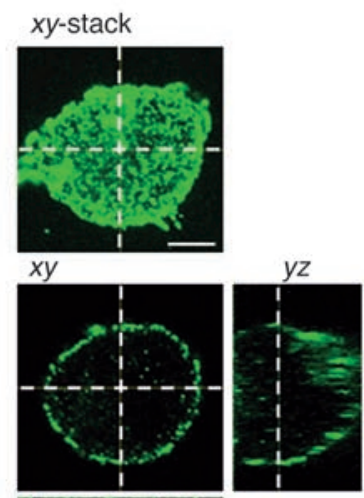

WT
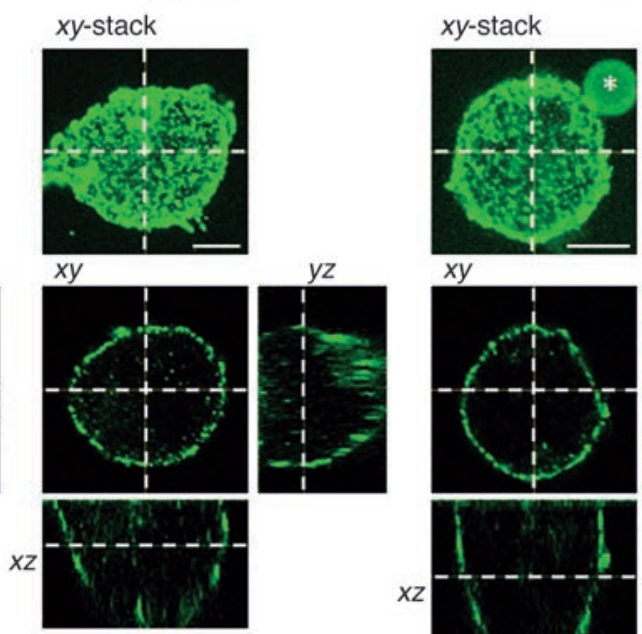

E

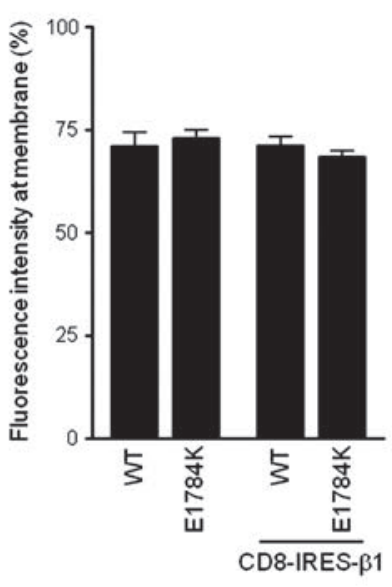

CD8-IRES- $\beta 1$

E1784K

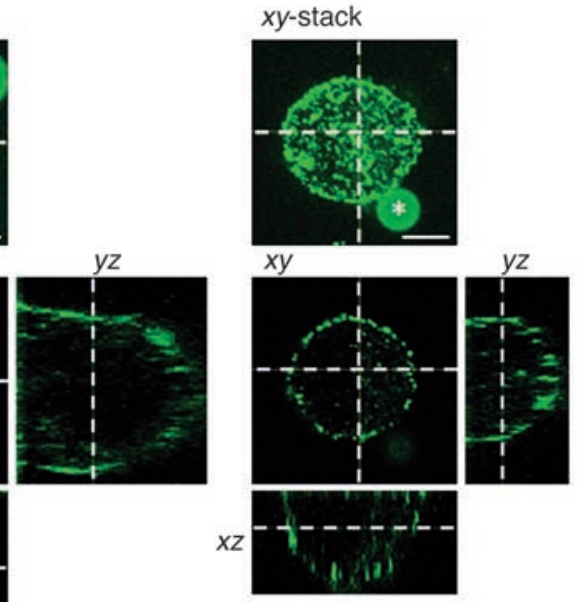

$x y$-stack

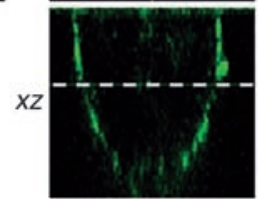

Figure 4

Gating and trafficking properties of E1784K. (A) The voltage dependence of steady-state fast inactivation and activation of E1784K, measured with standard pulse protocols shown in insets, were significantly shifted in the hyperpolarizing $(-15.0 \mathrm{mV})$ and depolarizing (+12.5 mV) directions, respectively. (B) Recovery from inactivation assessed by the double-pulse protocol was nearly identical between WT and E1784K. (C) Slow inactivation, measured by the double-pulse protocol shown in inset, was significantly enhanced in $\mathrm{E} 1784 \mathrm{~K}(P<0.05)$ in the magnitude of slow inactivation at all prepulse durations from $10 \mathrm{~ms}$ to $2,000 \mathrm{~ms}$. (D) Cells expressing either FLAG-tagged WT or E1784K in the presence or absence of pCD8-IRES- $\beta 1$ were immunostained with anti-FLAG antibody. Optical sections of Alexa Fluor 488 (green) were obtained using a confocal laser scanning microscope. Upper and lower panels show the $x y$-stacked image and the $x y$ image sliced at $z$ axis on the $x z$ and the $y z$ images, respectively. The $x z$ and $y z$ images indicate horizontal and vertical sections at the $x$ axis and $y$ axis on the $x y$ image. Asterisks and scale bars in $x y$-stacked images represent anti-CD8 Dynabeads and $5 \mu \mathrm{m}$, respectively. Note that both WT and E1784K predominantly localized to the plasma membrane regardless of $\beta 1$ subunit coexpression. (E) Membrane expression of Nav1.5 observed in $\mathbf{D}$ was quantified as described in Methods. Fluorescence intensity of Nav1.5 staining at plasma membrane, expressed as percentage relative to that of entire cell area, was comparable between WT and E1784K regardless of $\beta 1$ subunit coexpression. 
A

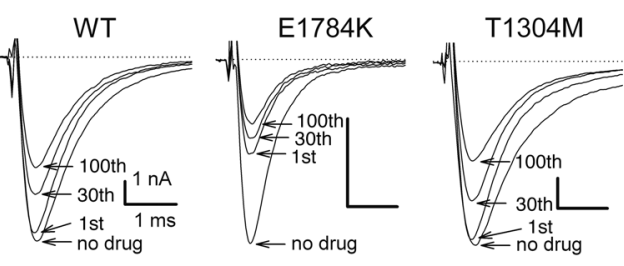

B

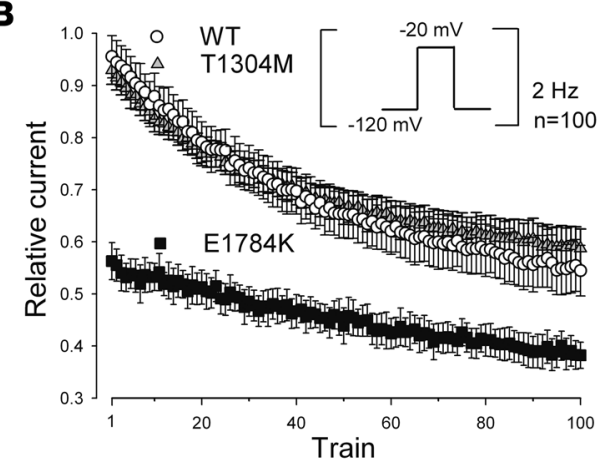

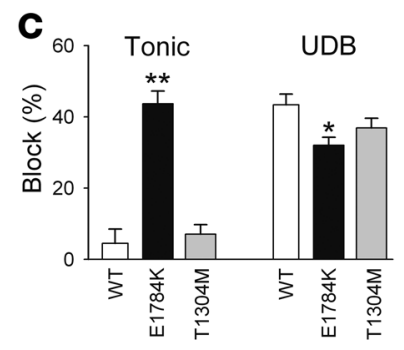

D

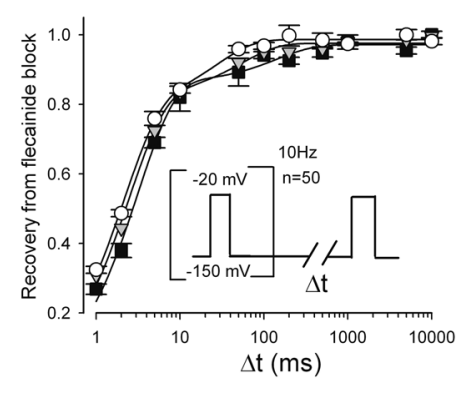

\section{Figure 5}

Tonic block and UDB by flecainide. (A) Representative current traces of WT, E1784K, and T1304M before and after $10 \mu \mathrm{mol} / \mathrm{l}$ flecainide. A train of 100 pulses (to $-20 \mathrm{mV}$ for $20 \mathrm{~ms}$ ) was applied at $2 \mathrm{~Hz}$ from a holding potential of $-120 \mathrm{mV}$. Numbers indicate the first, 30th, and 100th pulse of the $2-\mathrm{Hz}$ train. Zero current levels are indicated by dotted lines. (B) Time course of the peak current levels after application of $10 \mu \mathrm{mol} / \mathrm{I}$ flecainide. Peak current levels recorded with each pulse were normalized to the baseline prior to flecainide treatment. (C) Tonic block, determined by the first test pulse after application of flecainide, was weak in WT $(4.5 \% \pm 4.0 \%, n=5)$ and T1304M $(7.1 \% \pm 2.7 \%, n=5 ; P=\mathrm{NS})$ but was remarkably enhanced in E1784K $\left(43.7 \% \pm 8.0 \%, n=5 ;{ }^{* \star} P<0.001\right)$. Conversely, UDB, determined by the difference between first and 100 th pulses relative to the first pulse, was slightly attenuated in E1784K (WT, 43.4\% $\pm 3.0 \%$; E1784K, $32.0 \% \pm 2.2 \%$; $\left.{ }^{*} P<0.05\right)$, but not in T1304M $(36.9 \% \pm 2.7 \% ; P=N S)$. Net flecainide block at the 100th train was significantly enhanced in E1784K (WT, 45.5\% $\pm 5.0 \%$; E1784K, $61.8 \% \pm 2.5 \%$; $P<0.01)$ but not in T1304M (41.2\% $\pm 3.2 \% ; P=\mathrm{NS})$. (D) Recovery from flecainide block. Cells were held at a potential of $-150 \mathrm{mV}$ and superfused with $30 \mu \mathrm{mol} / \mathrm{l}$ flecainide, and a train of 50 pulses (to $-20 \mathrm{mV}$ for $20 \mathrm{~ms}$ ) at $10 \mathrm{~Hz}$ was applied to block Na channels. The pulse protocol cycle time was $60 \mathrm{~s}$. Recovery from block was then assessed by a $-20-\mathrm{mV}$ test pulse applied after varying the duration of a $-150-\mathrm{mV}$ repolarization period $(\Delta t)$. Peak current was normalized, and the data were fit to a double exponential function.

$-37.2 \pm 0.9 \mathrm{mV}, n=13 ; P<0.001)$. For example, conductance elicited by a depolarizing pulse of $-30 \mathrm{mV}$ to the maximum conductance was $0.93 \pm 0.05$ in WT channel but was only $0.66 \pm 0.02$ in E1784K channel. Both the negative shift of steady-state inactivation and the positive shift of activation reduced channel availability and conductance, respectively, and thus were predicted to decrease $\mathrm{Na}$ current during the upstroke of the action potential, the key features of BrS. Slope factors for inactivation were comparable (WT, $6.93 \pm 0.23 \mathrm{mV}$; E1784K, $7.09 \pm 0.12 \mathrm{mV}$ ), but slope factors of activation were significantly larger in E1784K (WT, $-6.08 \pm 0.26 \mathrm{mV}$; E1784K, $-8.06 \pm 0.20 \mathrm{mV} ; P<0.001)$, indicating that the activation in E1784K was less voltage sensitive (Figure 4A).

The proportion of channels available depends on the extent of recovery from inactivation. Recovery from fast inactivation was assessed by a standard double-pulse protocol using a recovery potential of $-120 \mathrm{mV}$, and there was no difference between WT and E1784K in the time constants for inactivation and the fractions of fast or slow recovery components (Figure 4B). Some mutant $\mathrm{Na}$ channels, including 1795insD (10) and T1620M (20), have been reported to display defects in the kinetics of or recovery from a "slower" inactivation process, sometimes referred to as $\mathrm{I}_{\mathrm{M}}$, and this defect is recognized as an important biophysical feature in some cases of $\mathrm{BrS}$ (21). This may explain the greater reduction in $\mathrm{Na}$ channel availability during excitation in LQT3 patients with 1795 insD (10) compared with those with $\triangle \mathrm{KPQ}$. We therefore ana- lyzed the onset of $\mathrm{I}_{\mathrm{M}}$ by varying prepulses of $1 \mathrm{~ms}$ to $2 \mathrm{~s}$ followed by a brief hyperpolarization to allow channels to recover from fast inactivation prior to a final test pulse. Double exponential curve fitting revealed that the amplitude of the fraction of the fast inactivating component $\left(\mathrm{A}_{\mathrm{f}}\right)$ was significantly larger in $\mathrm{E} 1784 \mathrm{~K}$ (WT, $0.12 \pm 0.02, n=10$; E1784K, $0.20 \pm 0.02, n=12 ; P<0.005$ ), and $\tau_{\mathrm{s}}$ was significantly smaller in E1784K (WT, $1883 \pm 669 \mathrm{~ms}$; E1784K, $554 \pm 101 \mathrm{~ms} ; P<0.05)$. Other parameters were comparable between WT and E1784K; $\left(\mathrm{A}_{\mathrm{s}}\right.$ : WT, $0.24 \pm 0.04$; E1784K, $0.19 \pm 0.01 ; P=\mathrm{NS} ; \tau_{\mathrm{f}}: \mathrm{WT}, 6.6 \pm 3.5 \mathrm{~ms} ; \mathrm{E} 1784 \mathrm{~K}, 1.3 \pm 0.2 ; P=\mathrm{NS}$; constant value $A_{\infty}$ : WT, $0.64 \pm 0.04 ; \mathrm{E} 1784 \mathrm{~K}, 0.60 \pm 0.01 ; P=\mathrm{NS}$ ). These data show that the extent of $\mathrm{I}_{\mathrm{M}}$ was significantly enhanced in E1784K similar to $1795 \mathrm{insD}(10)$, and provide a further mechanism for the reduced channel availability.

Another possible pathophysiological mechanism underlying the reduced peak Na current responsible for Brugada-type ST elevation in LQT3 is failure of mutant channel proteins to be properly trafficked to the cell surface $(22,23)$. To test this concept, an extracellular FLAG epitope was introduced in both WT and E1784K channels, and their cellular distribution was determined by a confocal laser scanning microscopy in tsA-201cells. As depicted in the $x z$ and $y z$ sections of Figure 4D, the distributions of anti-FLAG antibody were predominantly expressed in the plasma membrane and indistinguishable between WT and E1784K with or without coexpression of $\beta 1$ subunit (that has been implicated 

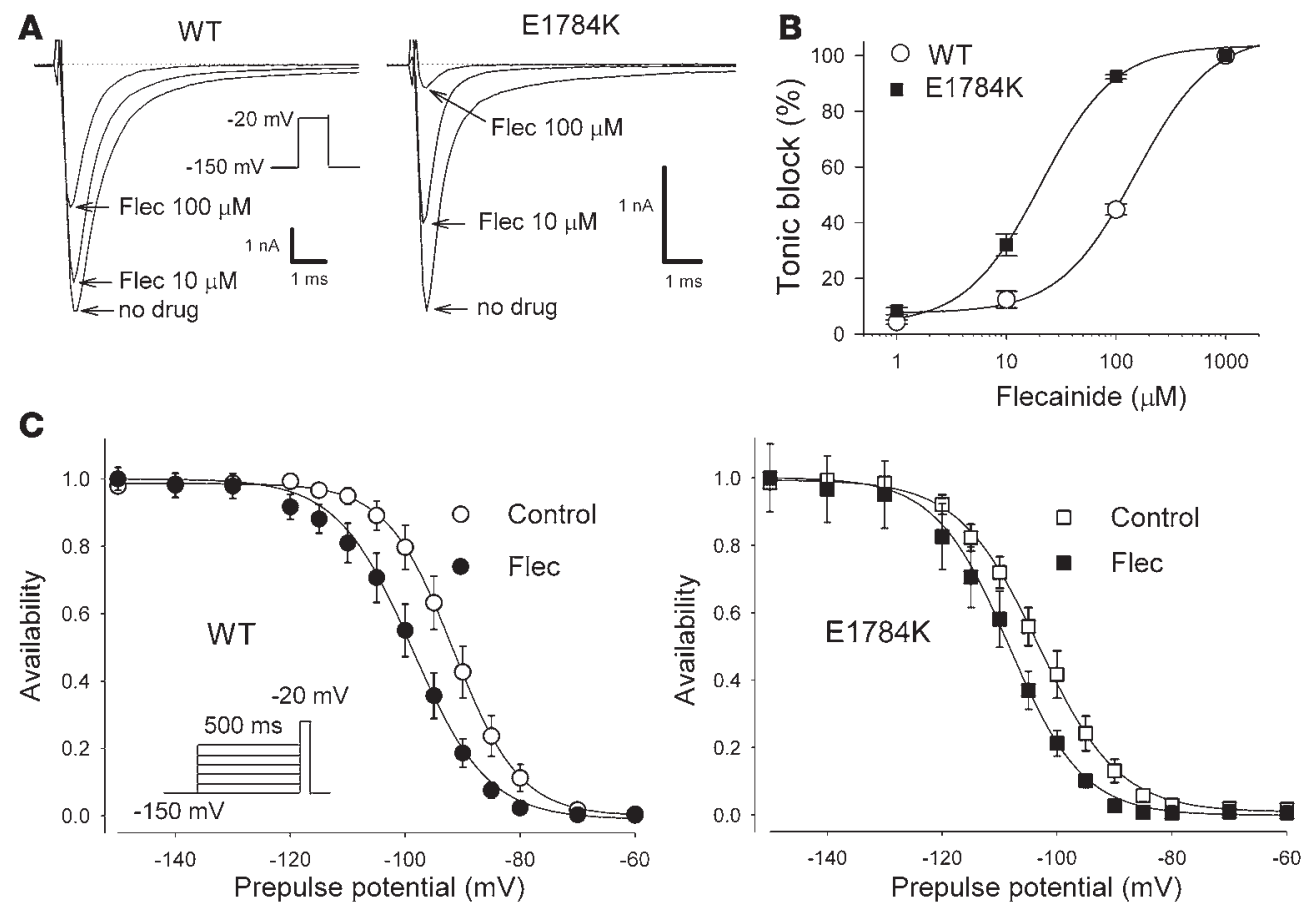

\section{Figure 6}

State-dependent flecainide block. (A) Representative steady-state current traces of WT and E1784K before and after flecainide (10 and $100 \mu \mathrm{mol} / \mathrm{l})$. Cells were depolarized by $-20 \mathrm{mV}$ from a holding potential of $-150 \mathrm{mV}$. Currents were normalized by the peak current before flecainide and were superimposed. Calibration bars indicate $1 \mathrm{nA}$ and $1 \mathrm{~ms}$. (B) Concentration-response curve for flecainide-induced tonic block in WT ( $n=5)$ and $\operatorname{E1784K}(n=5)$. The normalized peak currents were fit to the Hill equation $B(\%)=100 /\left(1+\left(D / / C_{50}\right)^{n H}\right)$, where $B$ is the percentage block at drug concentration $D$, and $n H$ is the Hill coefficient. The $I C_{50}$ values, representing dissociation constants for resting state $\left(K_{R}\right)$ were WT, $150.3 \mu \mathrm{mol} / \mathrm{I}$ and E1784K, $20.4 \mu \mathrm{mol} / \mathrm{l}$. Thus the mutant channel was far more sensitive to tonic block by flecainide. (C) Dissociation constant for inactivated channels. Cells were depolarized by prepulses ranging from $-150 \mathrm{mV}$ to $-60 \mathrm{mV}$ for $500 \mathrm{~ms}$ from a holding potential of $-150 \mathrm{mV}$ to ensure steady-state inactivation, followed by a $-20-\mathrm{mV}$ test pulse in the presence or absence of $30 \mu \mathrm{mol} / \mathrm{l}$ flecainide. The pulse protocol cycle time was $60 \mathrm{~s}$. Peak Na currents were normalized to the maximum Na current in the absence or presence of flecainide and were fit to the Boltzmann equation. The dissociation constant for the inactivated state $\left(K_{\mathrm{I}}\right)$ was calculated using Bean's equation (27): $V_{1 / 2}-V_{1 / 2 c}=k \times \ln \left(\left[1+D / K_{R}\right] /\left[1+D / K_{1}\right]\right)$, where $V_{1 / 2}$ and $V_{1 / 2 c}$ are the midpoints of steady-state inactivation for flecainide and control, respectively, $D$ is the flecainide concentration, and $K_{\mathrm{R}}$ is the dissociation constant for the resting state. The $K_{\mathrm{R}}$ values are equivalent to the $I_{50}$ values measured at a holding potential of $-150 \mathrm{mV}$ (WT, $150.3 \mu \mathrm{mol} / \mathrm{l} ; \mathrm{E} 1784 \mathrm{~K}, 20.4 \mu \mathrm{mol} / \mathrm{l})$ in B.

as a modulator of cell surface expression; ref. 24). Figure 4E shows the quantitative analysis of the fluorescent intensity in the membrane divided by the fluorescence intensity of entire cell area measured from 10 to 13 cells. Fluorescence intensity in the membrane was comparable between WT and E1784K regardless of the coexpressed $\beta 1$ subunit (WT, $71.5 \pm 3.5 \%, n=12$; E1784K, $72.9 \pm 2.0 \%$, $n=10 ; \mathrm{WT}+\beta 1,71.1 \pm 2.2 \%, n=13 ; \mathrm{E} 1784 \mathrm{~K}+\beta 1,68.4 \pm 1.6 \%$, $n=12 ; P=\mathrm{NS})$. Thus our immunocytochemical analyses provide strong evidence that the loss-of-function properties displayed by E1784K are most likely attributable to the observed changes in current gating rather than a change in channel density.

Molecular mechanisms of enhanced flecainide sensitivity. Class IC Na channel blockers generated the BrS phenotype in 5 of 9 patients challenged. We therefore investigated tonic block and use-dependent block (UDB) by flecainide in WT and E1784K channels expressed in tsA-201 cells, and compared them with those of T1304M, a mutation that did not show ST elevation during the flecainide challenge test (13). Cells transfected with WT, E1784K, or $\mathrm{T} 1304 \mathrm{M}$ were depolarized by $2-\mathrm{Hz}$ pulse trains at $-20 \mathrm{mV}$ for $20 \mathrm{~ms}$ from the holding potential of $-120 \mathrm{mV}$ in the absence or presence of $10 \mu \mathrm{mol} / 1$ flecainide. Under drug-free conditions, peak current reduction after the 100 pulses was less than $2 \%$ of the peak current for WT, E1784K, and T1304M. During exposure to $10 \mu \mathrm{mol} / \mathrm{l}$ flecainide, peak currents normalized to predrug baseline were progressively reduced by the repetitive pulses (Figure 5, A and B). There was a remarkable difference in the extent of first pulse (tonic) block that was only $4.5 \% \pm 4.0 \%$ for WT $(n=5)$, and $7.1 \% \pm 2.7 \%$ for $\mathrm{T} 1304 \mathrm{M}(n=5, P=\mathrm{NS})$, compared with substantial tonic block in E1784K $(43.7 \% \pm 8.0 \%$, $n=5, P<0.001)$. Conversely, UDB, determined by the difference in peak current values between the first and hundredth test pulses relative to the first pulse, was slightly attenuated in E1784K (WT, 43.4\% $\pm 3.0 \%$; E1784K, 32.0\% $\pm 2.2 \%$; $P<0.05$ ), but not in $\mathrm{T} 1304 \mathrm{M}(36.9 \% \pm 2.7 \% ; P=\mathrm{NS})$ (Figure $5 \mathrm{C})$. The net effect of flecainide after a train of 100 pulses was significantly greater in E1784K than WT (WT, $45.5 \% \pm 5.0 \%$; E1784K, $61.8 \% \pm 2.5 \% ; P<0.001)$ but not in T1304M (41.2\% $\pm 3.2 \%$; $P=\mathrm{NS})$. This attenuated flecainide UDB in E1784K is in striking contrast to that reported for $1795 \mathrm{insD}(12)$ and $\triangle \mathrm{KPQ}(25)$, which showed enhancement in both tonic and use-dependent flecainide block. UDB is profoundly affected by the recovery from drug block, and delayed recovery from flecainide block has been 


\section{Table 1}

Reported biophysical properties of LQT3 mutations

\begin{tabular}{|c|c|c|c|c|c|}
\hline & \multicolumn{5}{|c|}{ SCN5A mutations } \\
\hline & 1795insD & $\triangle K P Q$ & $\Delta K 1500$ & E1784K & $\mathrm{T} 1304 \mathrm{M}$ \\
\hline \multicolumn{6}{|l|}{ Clinical features } \\
\hline QT prolongation & + & + & + & + & + \\
\hline ST elevation ${ }^{A}$ & + & + & + & + & - \\
\hline Sinus node dysfunction & + & + & + & + & - \\
\hline \multicolumn{6}{|c|}{ Biophysical and pharmacological properties } \\
\hline Persistent Na current & + & + & + & + & + \\
\hline Shift of $V_{1 / 2}$ (inactivation) & Negative & Negative & Negative & Negative & Positive \\
\hline Shift of $V_{1 / 2}$ (activation) & Positive & Positive & Positive & Positive & Positive \\
\hline Current decay & Slower & Faster & $\leftrightarrow$ & Faster & Faster \\
\hline Recovery from inactivation & Slower & Faster & ND & $\leftrightarrow$ & Faster \\
\hline Slow inactivation & Enhanced & $\leftrightarrow$ & ND & Enhanced & $\leftrightarrow$ \\
\hline Tonic block by flecainide & Enhanced & Enhanced & Enhanced & Enhanced & $\leftrightarrow$ \\
\hline UDB by flecainide & Enhanced & Enhanced & ND & Diminished & $\leftrightarrow$ \\
\hline References & $10,11,28$ & 2,25 , this study & 15 & 17 , this study & 29 , this study \\
\hline
\end{tabular}

ASpontaneous or Na channel blocker-induced ST elevation. $\leftrightarrow$, comparable with WT; ND, not determined.

implicated in the augmented flecainide sensitivity in $1795 \mathrm{insD}$ (12) and $\triangle \mathrm{KPQ}$ (25). Accordingly, we evaluated recovery from flecainide block using the protocol shown in the inset of Figure 5D. Cells were initially subjected to 50 pulses ( $-20 \mathrm{mV}$ for $20 \mathrm{~ms}$ ) from a holding potential of $-150 \mathrm{mV}$ at $10 \mathrm{~Hz}$ in the presence of $30 \mu \mathrm{mol} / 1$ flecainide, followed by a variable recovery time $(\Delta t)$ and a test pulse of $-20 \mathrm{mV}$. Recovery from flecainide block was virtually identical among all 3 channels, compatible with the observation that the drug-free recovery from inactivation of WT and E1784K were comparable (Figure 4B). Taken together, these results indicate that the $\mathrm{E} 1784 \mathrm{~K}$ channels are much more sensitive to block by flecainide than are the WT and T1304M channels, and that this augmented sensitivity is attributable to enhanced tonic block rather than a change in UDB.

If the extent of tonic block roughly parallels the voltage dependence of availability of drug-free channels, then the difference between WT and E1784K should be attenuated when cells are depolarized from a very negative holding potential, where the channels will be similarly available (Figure 4A). At a holding potential of $-150 \mathrm{mV}$, the magnitude of flecainide tonic block was diminished in both WT and E1784K, but tonic block at $-150 \mathrm{mV}$ was still greater in E1784K (Figure 6A). Dose-response curves for flecainide block measured at a holding potential of $-150 \mathrm{mV}$ (thus representing drug affinity for the resting state) are shown in Figure $6 \mathrm{~B}$. The E1784K channels were 7.5 -fold more sensitive to resting-state block by flecainide than were the WT channels $\left(I C_{50}\right.$ : WT, $150.3 \mu \mathrm{mol} / \mathrm{l}$; E1784K, $20.4 \mu \mathrm{mol} / \mathrm{l})$.

The diminished UDB in E1784K may be attributable to reduced flecainide affinity for inactivated channels. To test this idea, flecainide affinity for the inactivated state was measured as previously described (26). Steady-state channel availability was determined in the presence or absence of $30 \mu \mathrm{mol} / 1$ flecainide by using $500 \mathrm{~ms}$ prepulses ranging from -150 to $-60 \mathrm{mV}$ to ensure a steady state before the test pulse (Figure 6C). The apparent dissociation constant for the inactivated channels $\left(K_{\mathrm{I}}\right)$ was calculated using Bean's equation (27). $K_{\mathrm{I}}$ values for flecainide of WT and E1784K were comparable (WT, $7.1 \mu \mathrm{mol} / 1$; E1784K, $5.4 \mu \mathrm{mol} / \mathrm{l}$ ). These results suggest that the enhanced sensitivity to flecainide of the E $1784 \mathrm{~K}$ is explained mainly by gating-dependent mechanisms of the mutant channel (i.e., enhanced inactivation), but increased drug affinity to resting-state channels may also be involved.

Spectrum of SCN5A dysfunction in LQT3 associated with BrS and sinus node dysfunction. There are several possible loss-of-function properties of the $\mathrm{Na}$ channel that have been linked to the BrS phenotype. These include a negative shift of steady-state inactivation (which includes enhanced closed-state inactivation) (12), a positive shift of activation, faster current decay, enhanced slow inactivation (20), slower recovery from inactivation, a trafficking defect of the channel molecule (22), and enhanced sensitivity to Na channel blockers.

To explore the functional determinants for the phenotypic overlap of BrS in LQT3 patients, we compared the biophysical and pharmacological properties of reported LQT3 mutations and sought features commonly and specifically observed in those manifesting a BrS phenotype (Table 1). The overlapping phenotype (LQT3 and $\mathrm{BrS})$ has been previously reported for $1795 \mathrm{insD}(10,11), \Delta \mathrm{KPQ}$ $(13,14), \Delta \mathrm{K} 1500$ (15), and E1784K (13). In contrast, a carrier of T1304M did not show ST elevation during a flecainide test (13). Similarly, sinus node dysfunction has been reported in carriers of the same SCN5A mutations, $1795 \mathrm{insD}(28), \triangle \mathrm{KPQ}(16), \triangle \mathrm{K} 1500$ (15), E1784K (17), and D1790G (7), but not in other SCN5A mutations, including T1304M. Thus, it is plausible to speculate that the biophysical characteristics common to these mutations but not found in T1304M are channel properties responsible for evoking mixed phenotypes of $\mathrm{BrS}$ and sinus node dysfunction in patients with LQT3. To this end, Table 1 compares the functional properties of E1784K and those reported for $1795 \mathrm{insD}, \Delta \mathrm{KPQ}, \Delta \mathrm{K} 1500$, $\mathrm{E} 1784 \mathrm{~K}$, and T1304M (29). Because inconsistency with the gating properties of $\triangle \mathrm{KPQ}$ has been noted among several reports, we reevaluated this mutant under conditions identical to those we used for E1784K. Consistent with the initial report by Bennett et al. (2), steady-state inactivation of $\triangle K P Q$ was significantly shifted in the negative direction by $-8.9 \mathrm{mV}\left(V_{1 / 2}=-95.7 \pm 1.1 \mathrm{mV}, n=7\right)$ compared with WT, and activation was significantly shifted in the positive direction $\left(V_{1 / 2}=-34.9 \pm 0.9 \mathrm{mV}, n=7\right)$, similar to E1784K. Among the biophysical properties listed in Table 1, we found that both the negative shift in steady-state inactivation and 
the enhanced tonic block by flecainide are common to $1795 \mathrm{insD}$, $\triangle \mathrm{KPQ}, \Delta \mathrm{K} 1500$, and $\mathrm{E} 1784 \mathrm{~K}$, but not to T1304M. This negative shift of inactivation will reduce the availability of the channels at the resting membrane potential and increase the proportion of inactivated channels in both the open and closed states, reducing $\mathrm{Na}$ current and increasing the sensitivity to $\mathrm{Na}$ channel blockers. A positive shift in activation is another loss-of-function property evident in all the mutants including T1304M, making it less likely that this specific channel property underlies mixed clinical phenotypes in LQT3. Other channel properties such as current decay, recovery from inactivation, slow inactivation, or UDB were not common among 1795 ins D, $\triangle \mathrm{KPQ}, \Delta \mathrm{K} 1500$, and E1784K.

\section{Discussion}

The main findings of this study are that the SCN5A mutation, E1784K, was found in 34\% of LQT3 probands and showed overlapping clinical phenotypes of $\mathrm{BrS}$ and sinus node dysfunction in patients from different genetic backgrounds. In vitro functional characterization of this mutation and comparison to properties reported for other LQT3 variants suggest that a negative shift of steady-state $\mathrm{Na}$ channel inactivation and enhanced tonic block in response to $\mathrm{Na}$ channel blockers confer an additional $\mathrm{BrS} /$ sinus node dysfunction phenotype.

E1784K was originally reported by Wei et al. (17) and subsequently by several other groups $(13,30-32)$. Other groups have also indicated that this is a common LQT3 mutation. Napolitano et al. previously reported that E1784K is a "non-private" LQT3 mutation (i.e., that it occurred more than once in their LQTS population) (33). Similarly, in genetic screening of 541 unrelated LQTS patients, Tester et al. demonstrated that E1784K was the most prevalent LQT3 mutation $(4 / 26,15.4 \%)(32)$.

$\mathrm{Na}$ channel blocker therapy is widely used in LQT3 because of block of persistent $\mathrm{Na}$ current during repolarization (34). However, these agents, especially class IC drugs, also unmask or exacerbate ST elevation in BrS. Despite this apparent divergence of $\mathrm{Na}$ channel blocker action, some LQT3 patients display ST elevation characteristic of BrS spontaneously (10-12) or during class IC drug therapy $(13,14)$. The results of the present study indicate that the BrS/LQT3 overlap is not likely to reflect coinherited genetic variations, gender, or ethnicity and thus implicate the biophysical properties of specific mutant channels as the primary mediator of this mixed phenotype. Furthermore, this overlapping phenotype appears to be more common than generally recognized because it is observed in E1784K (a prevalent mutation), as well as other LQT3 mutations (1795insD, $\triangle \mathrm{KPQ}, \Delta \mathrm{K} 1500)$. These observations have raised a concern about the safety of class IC drug therapy in LQT3 patients (13) and prompted us to examine in more detail the underlying mechanisms of drug-induced block in $\mathrm{Na}$ channels with these mutations.

The mutation 1795 insD (10-12), which produces an overlapping phenotype, generates a wide range of biophysical abnormalities, including a negative shift in inactivation, enhanced slow inactivation, and delayed recovery from inactivation in addition to the hallmark LQT3 abnormality of non-inactivating persistent $\mathrm{Na}$ current (12). This negative shift of steady-state inactivation, which is compatible with enhanced closed-state inactivation, tends to increase the proportion of the inactivated channels at the resting potential and thus augments tonic block by flecainide. This property, as well as increased persistent current, is commonly observed in the 3 other LQT3 mutations associated with BrS and sinus node dysfunction (E1784K, $\Delta \mathrm{KPQ}$, and $\Delta \mathrm{K} 1500$ ). However, T1304M, which is not associated with $\mathrm{BrS}$ or sinus node dysfunction, shows a positive shift of steady-state inactivation (Table 1).

Enhanced slow inactivation and UDB, which are thought to underlie the phenotype produced by T1620M (20), a "BrS-only" (non-LQT3) mutation, have also been reported with $1795 \mathrm{insD}$ (12). Although slow inactivation was enhanced in E1784K (Figure 4C), and UDB was enhanced in $\triangle \mathrm{KPQ}$ (25), these characteristics are not common among the 4 mutations with LQT3/BrS overlap. Indeed, UDB by flecainide was diminished in E1784K (Figure 5, A-C). These findings suggest that slow inactivation or use-dependent inactivation is not the principal mechanism for the BrS-type ST elevation in LQT3.

In addition to right precordial ST elevation, a secondary phenotype of sinus node dysfunction has been reported in 4 SCN5A mutations (E1784K, 1795insD, $\triangle \mathrm{KPQ}$, and $\Delta \mathrm{K} 1500)$. Although the functional relevance of $\mathrm{Na}$ channels in sinus nodal pacemaker function has been debated, there is evidence for Na current in the sinus node periphery (35), and other TTX-sensitive Na channels may contribute (36). Patch clamp and computer simulation studies of $1795 \mathrm{insD}$ have demonstrated that the negative shift of inactivation reduces $\mathrm{Na}$ current during diastolic depolarization; also, the persistent $\mathrm{Na}$ current increases action potential duration, leading to a slowing of sinus rate (28). A negative shift in inactivation is observed in $\mathrm{E} 1784 \mathrm{~K}, 1795 \mathrm{insD}, \Delta \mathrm{KPQ}$, and $\Delta \mathrm{K} 1500$ and may play a role in the overlap of the LQT3 clinical phenotype with BrS and sinus node dysfunction in the mutation carriers. In summary, a negative shift in inactivation and enhanced tonic block are common biophysical properties observed among SCN5A mutations with the LQT3/BrS overlapping phenotype. These findings suggest that prophylactic class IC drugs should be avoided in LQT3 mutations displaying these biophysical properties in vitro.

Because this was a multicenter study, the Na channel blockers used for provocation testing were not the same. In addition, 4 patients challenged with flecainide did not display right precordial ST elevation; this negative flecainide test in 1 individual could have been due to variable efficacy of $\mathrm{Na}$ channel blockers or day-to-day variability in autonomic tone (37). While a negative shift in steady-state inactivation appears to be a common biophysical property among LQT3 mutations (including E1784K) displaying the mixed phenotype, the number of LQT3 mutations that have been evaluated in this detail is still small. Moreover, biophysical and pharmacological properties presented in tsA-201 cells may not necessarily reflect the situation in vivo, and the effects of the mutation may be different in ventricular myocytes compared with sinus node cells. Thus, further studies that combine clinical and in vitro phenotyping in LQT3 mutations with and without overlapping clinical phenotypes will be required to confirm the findings of the present study.

E1784K is the most common LQT3 mutation. In patients with this and other LQT3 mutations, overlap with BrS and sinus node dysfunction is relatively common. Our in vitro studies with E1784K and previous reports in LQT3 mutations with and without this clinical overlap syndrome implicate a negative shift in inactivation and enhanced tonic block by drugs as underlying mechanisms. These data suggest that patients with LQT3 mutations displaying these characteristics in vitro should not receive class IC drugs. Furthermore, the present findings reinforce the general concept that in vitro characterization of the function of ion channel variants is a key component in generating specific therapeutic strategies for patient management. 


\section{Methods}

\section{Clinical evaluation}

The study population included 44 genotyped LQT3 families with different ethnic backgrounds (20 Asian, 24 Caucasian) ascertained from a total of 7 institutions (Japan, $n=3$; USA, England, Italy, and Germany, $n=1$ each). In these families, E1784K (c. 5350C $\rightarrow \mathrm{A}$ ) was the most prevalent SCN5A mutation $(n=15 ; 34 \%)$. The mean age of the probands ( 10 men, 5 women) was $22 \pm 11$ years at diagnosis (mean $\pm \mathrm{SD}$, ranging from 7 to 43 years). Two probands died suddenly (A;II:6 and C;III:1; Figure 1), one at rest and the other whilst driving a car. In both cases, no ante mortem data was available and an autopsy could not establish a cause of death. The 15 families were comprised of 93 surviving members, of whom 66 underwent genetic testing. Family members underwent physical examination, ECG, exercise stress test, and Holter recording. The diagnosis of LQTS in the probands, or in the family members if probands died suddenly before evaluation, was established using the revised criteria suggested by Schwartz (8). Na channel blocker challenge was conducted using mexiletine $(2 \mathrm{mg} / \mathrm{kg})$, flecainide (2 mg/kg), ajmaline (1 mg/kg), or pilsicainide $(1 \mathrm{mg} / \mathrm{kg})$ administered intravenously, with positive (coved) and negative responses determined using standard 12-lead ECGs, as previously described (38).

Sinus node dysfunction was considered if one of the following conditions was recorded at one or more occasions when inappropriate for the circumstances: (a) sinus bradycardia, (b) sinus arrest or exit block, and (c) combinations of sinoatrial and atrioventricular conduction disturbances; these bradyarrhythmias often occurred in conjunction with paroxysmal atrial tachyarrhythmias (39).

\section{Genetic analysis}

All probands and family members who participated in the study gave written informed consent before the genetic and clinical investigations, in accordance with the standards of the Declaration of Helsinki and local ethics committees. Genetic analysis was performed on genomic DNA extracted from peripheral white blood cells using standards methods. Coding regions of KCNQ1, KCNH2, SCN5A, KCNE1, and KCNE2 were amplified by PCR using exon-flanking intronic primers $(31,40)$, and direct DNA sequencing was performed using ABI 310, 3130, and 3730 genetic analyzers (Applied Biosystems) (17). In some cases, denaturing high-performance liquid chromatography with the Transgenomic Wave Instrument (Transgenomic Inc.) was applied for the screening of polymorphisms prior to direct sequencing.

Construction of expression plasmids and transfection of mammalian cell lines. A $0.5-\mathrm{kb}$ HindIII/XhoI fragment ( $5^{\prime}$ cloning site - nt. 533) and a 1.6-kb BstEII/XbaI fragment (nt. 4626 - 3' cloning site) of WT human heart $\mathrm{Na}$ channel $\alpha$ subunit (Nav1.5) were subcloned into the pBluescript II $\mathrm{SK}^{+}$plasmid (Stratagene) for the mutagenesis to generate the FLAGtagged channel and E1784K, respectively. Site-directed mutagenesis was performed by QuikChange site-directed mutagenesis kit (Stratagene) according to the manufacturer's instructions. A FLAG-tagged Nav1.5 plasmid was constructed by inserting the FLAG (DYKDDDK) epitope in frame at the extracellular loop of the domain 1 between Pro154 and Pro155. The FLAG-tag did not change the current densities or the gating properties of Nav1.5 (data not shown). Final constructs were assembled in the mammalian expression plasmid pcDNA3.1+ (Invitrogen) and then sequenced to verify creation of mutation and exclude polymerase errors. The plasmids encoding $\triangle \mathrm{KPQ}$ and $\mathrm{T} 1304 \mathrm{M}$ were constructed as previously described $(2,29)$. The human cell line tsA-201 was transiently transfected with WT, E1784K $\triangle \mathrm{KPQ}$, or T1304M plasmid using Lipofectamine (Invitrogen) or SuperFect (Qiagen) in combination with a bicistronic plasmid (pCD8-IRES-h $\beta_{1}$ ) encoding CD8 and the human Na channel $\beta_{1}$ subunit $\left(h \beta_{1}\right)$ to visually identify cells expressing heterologous $h \beta_{1}$ with Dynabeads M-450 CD8 (Dynal) (41). Electrophysiological measurements were performed $24-72 \mathrm{~h}$ after transfection.

Electrophysiology and data analysis. Na currents were recorded using the whole-cell patch clamp technique as previously described (41). Electrode resistance ranged from 0.8 to $1.5 \mathrm{M} \Omega$. Data acquisition was carried out using an Axopatch 200B patch clamp amplifier and pCLAMP8 software (Axon Instruments). Currents were filtered at $5 \mathrm{kHz}$ (-3 dB; 4-pole Bessel filter) and digitized using an analog-to-digital interface (Digidata 1322A; Axon Instruments). Experiments were carried out at room temperature $\left(20-22^{\circ} \mathrm{C}\right)$. Voltage errors were minimized using series resistance compensation (generally $80 \%$ ). Cancellation of the capacitance transients and leak subtraction were performed using an online $\mathrm{P} / 4$ protocol. The pulse protocol cycle time was $10 \mathrm{~s}$ unless otherwise stated. The data were analyzed using Clampfit 10 (Axon Instruments) and SigmaPlot 9 (SPSS Science). The holding potential was $-120 \mathrm{mV}$ unless otherwise stated. The bath solution contained (in mmol/l): $145 \mathrm{NaCl}, 4 \mathrm{KCl}, 1.8 \mathrm{CaCl}_{2}, 1 \mathrm{MgCl}_{2}, 10$ HEPES, and 10 glucose, $\mathrm{pH} 7.35$ (adjusted with $\mathrm{NaOH}$ ). The pipette solution (intracellular solution) contained (in mmol/l): $10 \mathrm{NaF}, 110 \mathrm{CsF}, 20 \mathrm{CsCl}, 10 \mathrm{EGTA}$, and 10 HEPES, pH 7.35 (adjusted with $\mathrm{CsOH}$ ). The time from establishing the whole-cell configuration to onset of recording was consistent cell-to-cell to exclude the possible time-dependent shift of steady-state inactivation.

To determine activation parameters, the current-voltage relationship was fit to the Boltzmann equation $I=\left(V-V_{\text {rev }}\right) \times G_{\max } \times\left(1+\exp \left[V-V_{1 / 2}\right] / k\right)^{-1}$, where $I$ is the peak Na current during the test pulse potential $V$. The parameters estimated by the fitting are $V_{\text {rev }}$ (reversal potential), $G_{\max }$ (maximum conductance), and $k$ (slope factor). Steady-state availability for fast inactivation was measured with a standard double-pulse protocol (Figure $4 \mathrm{~A}$, inset $)$, and the data were fit with the Boltzmann equation $I / I_{\max }=(1+$ $\left.\exp \left[\left(V-V_{1 / 2}\right) / k\right]\right)^{-1}$, where $I_{\max }$ is the maximum peak Na current, to determine the membrane potential for $V_{1 / 2}$ and $k$. Macroscopic current decay was analyzed by fitting data with a double exponential equation: $I / I_{\max }=A_{\infty}+A_{\mathrm{f}} \times \exp \left(-t / \tau_{\mathrm{f}}\right)+A_{\mathrm{s}} \times \exp \left(-t / \tau_{\mathrm{s}}\right)$, where $A_{\infty}$ and $t$ are constant value and time, respectively; $A_{\mathrm{f}}$ and $A_{\mathrm{s}}$ are fractions of fast and slow inactivating components, respectively; and $\tau_{\mathrm{f}}$ and $\tau_{\mathrm{s}}$ are time constants of $A_{\mathrm{f}}$ and $A_{s}$, respectively. Recovery from inactivation was assessed by the double-pulse protocol (Figure $4 \mathrm{~B}$, inset) and was analyzed by fitting the data to a double exponential equation: $I / I_{\max }=A_{\infty}+A_{\mathrm{f}} \times \exp \left(-t / \tau_{\mathrm{f}}\right)+A_{\mathrm{s}}$ $\times \exp \left(-t / \tau_{s}\right)$. The onset of slow inactivation was measured by the doublepulse protocol shown in the inset of Figure 4C. A brief repolarization pulse was applied to allow most channels to recover from fast inactivation, and the data were fit with biexponential functions.

In some experiments, cells were superfused with bath solution containing flecainide or TTX to determine their pharmacological effects. During the superfusion, cells were held at $-120 \mathrm{mV}$ unless otherwise stated. Tonic block was defined as a diminution in the peak Na current of the first depolarizing pulse after the exposure to the drug compared with that in the control, and UDB as a diminution in the peak Na current of the hundredth pulse compared with that of the first pulse after the drug. Flecainide (acetate salt) was made up in a $10 \mathrm{mmol} / \mathrm{l}$ stock solution and diluted in a bath solution, and data were recorded after 3-min periods for drug equilibration. All the chemicals were purchased from Sigma-Aldrich or Wako, except for flecainide and mexiletine, which were gifts from Eisai and Nippon Boehringer Ingelheim, respectively.

Immunocytochemistry. TsA-201 cells were transfected with the pcDNA3.1 $1^{+}$Nav1.5 plasmid encoding either WT or E1784K together with or without $\mathrm{pCD} 8$-IRES-h $\beta 1$. Two days later, cells were incubated with Dynabeads M-450 CD8 (Dynal) for $30 \mathrm{~min}$ at $37^{\circ} \mathrm{C}$ to identify the cells expressing both $\mathrm{h} \beta 1$ and $\mathrm{CD} 8$. The cells were then washed with PBS $(\mathrm{pH}$ 7.4), fixed in PBS containing $2 \%$ formaldehyde for $30 \mathrm{~min}$ at $4{ }^{\circ} \mathrm{C}$, and 
permeabilized with $0.05 \%$ Triton $\mathrm{X}-100$ for $30 \mathrm{~min}$ at $4^{\circ} \mathrm{C}$. After blocking with PBS containing $4 \%$ BSA for $1 \mathrm{~h}$ at room temperature, the cells were stained with anti-FLAG M2 monoclonal antibody (Sigma-Aldrich) for $1 \mathrm{~h}$ at room temperature. Protein reacting with antibody was visualized with Alexa Fluor 488-labeled secondary antibody (Molecular Probes). Optical sections of Alexa Fluor 488 were obtained with a FluoView FV1000 confocal laser scanning microscope (Olympus Corp.) with a $\times 60$ oil immersion objective lens. To quantify the membrane expression of Nav1.5, fluorescence intensity at the entire cell area and the plasma membrane region in the middle $x y$ image of $z$ series stack was measured using MetaMorph 6.1 software (Molecular Devices).

Statistics. Results are presented as means \pm SEM unless otherwise stated, and statistical comparisons were made using ANOVA with the Dunnett post-hoc test to evaluate the significance of differences between means. Statistical significance was assumed for $P<0.05$.

\section{Acknowledgments}

This work was supported in part by grants from MEXT Japan (18590757 to N. Makita and 14370225, 16209025, and 19390212 to M. Horie); the Ministry of Health, Labour and Welfare, Japan (H18-Research on Human Genome-002 to N. Makita, W. Shi- mizu, and M. Horie; Research on Nanotechnical Medical, H19nano-006 to N. Mochizuki); the Uehara Memorial Foundation (to W. Shimizu); the Program for the Promotion of Fundamental Studies in Health Sciences of the National Institute of Biomedical Innovation (to N. Mochizuki); the US Public Health Service (HL49989 and HL46681 to D.M. Roden, HL65962 to A.L. George Jr. and D.M. Roden, and HL33843 to L. Crotti and P.J. Schwartz); the Collaborative Research Effort (SFB, project C1) of the DFG (to E. Schulze-Bahr); the DFG (DFG Schu1082/3-1 and 3-2 to E. Schulze-Bahr); and the Fondation Leducq (to E. Schulze-Bahr). We thank M. Fukuoka, K. Kawai, and N. Ohashi for technical assistance.

Received for publication September 25, 2007, and accepted in revised form February 27, 2008.

Address correspondence to: Naomasa Makita, Department of Cardiovascular Medicine, Hokkaido University Graduate School of Medicine, Kita-15, Nishi-7, Kita-Ku, Sapporo 060-8638, Japan. Phone: 81-11-706-6973; Fax: 81-11-706-7874; E-mail: makitan03@ybb.ne.jp.
1. Keating, M.T. 1996. The long QT syndrome. A review of recent molecular genetic and physiologic discoveries. Medicine. 75:1-5.

2. Bennett, P.B., Yazawa, K., Makita, N., and George, A.L., Jr. 1995. Molecular mechanism for an inherited cardiac arrhythmia. Nature. 376:683-685.

3. Chen, Q., et al. 1998. Genetic basis and molecular mechanism for idiopathic ventricular fibrillation. Nature. 392:293-296.

4. Schott, J.J., et al. 1999. Cardiac conduction defects associate with mutations in SCN5A. Nat. Genet. 23:20-21.

5. Benson, D.W., et al. 2003. Congenital sick sinus syndrome caused by recessive mutations in the cardiac sodium channel gene (SCN5A). J. Clin. Invest. 112:1019-1028.

6. Schwartz, P.J., et al. 1995. Long QT syndrome patients with mutations of the SCN5A and HERG genes have differential responses to $\mathrm{Na}^{+}$ channel blockade and to increases in heart rate. Implications for gene-specific therapy. Circulation. 92:3381-3386.

7. Benhorin, J., et al. 2000. Effects of flecainide in patients with new SCN5A mutation: Mutationspecific therapy for long-QT syndrome? Circulation. 101:1698-1706.

8. Schwartz, P.J. 2006. The congenital long QT syndromes from genotype to phenotype: clinical implications. J. Intern. Med. 259:39-47.

9. Antzelevitch, C. 2001. The Brugada syndrome: ionic basis and arrhythmia mechanisms. J. Cardiovasc. Electrophysiol. 12:268-272.

10. Veldkamp, M.W., et al. 2000. Two distinct congenital arrhythmias evoked by a multidysfunctional $\mathrm{Na}^{+}$channel. Circ. Res. 86:E91-E97.

11. Bezzina, C., et al. 1999. A single $\mathrm{Na}^{+}$channel mutation causing both long-QT and Brugada syndromes. Circ. Res. 85:1206-1213.

12. Viswanathan, P.C., et al. 2001. Gating-dependent mechanisms for flecainide action in SCN5A-linked arrhythmia syndromes. Circulation. 104:1200-1205.

13. Priori, S.G., et al. 2000. The elusive link between LQT3 and Brugada syndrome: The role of flecainide challenge. Circulation. 102:945-947.

14. Moss, A.J., et al. 2005. Safety and efficacy of flecainide in subjects with long QT-3 syndrome ( $\triangle \mathrm{KPQ}$ Mutation): A randomized, double-blind, placebo-controlled clinical trial. Ann. Noninvasive Electrocardiol. 10:59-66.

15. Grant, A.O., et al. 2002. Long QT syndrome, Bru- gada syndrome, and conduction system disease are linked to a single sodium channel mutation. J. Clin. Invest. 110:1201-1209.

16. Moss, A.J., et al. 1995. ECG T-wave patterns in genetically distinct forms of the hereditary long QT syndrome. Circulation. 92:2929-2934.

17. Wei, J., et al. 1999. Congenital long-QT syndrome caused by a novel mutation in a conserved acidic domain of the cardiac $\mathrm{Na}+$ channel. Circulation. 99:3165-3171.

18. Berg, M.P., et al. 2001. Possible bradycardic mode of death and successful pacemaker treatment in a large family with features of long QT syndrome type 3 and Brugada syndrome. J. Cardiovasc. Electrophysiol. 12:630-636.

19. Schwartz, P. 1980. The long QT syndrome. In Sudden death. H. Kulbertus and H. Wellens, editors. Springer-Verlag. New York, New York, USA. 358-378.

20. Wang, D.W., Makita, N., Kitabatake, A., Balser, J.R., and George, A.L., Jr. 2000. Enhanced $\mathrm{Na}^{+}$channel intermediate inactivation in Brugada syndrome. Circ. Res. 87:E37-E43.

21. Balser, J.R. 2001. The cardiac sodium channel: gating function and molecular pharmacology. J. Mol. Cell. Cardiol. 33:599-613.

22. Baroudi, G., et al. 2001. Novel mechanism for Brugada syndrome: Defective surface localization of an SCN5A mutant (R1432G). Circ. Res. 88:e78-e83.

23. Liu, K., Yang, T., Viswanathan, P.C., and Roden, D.M. 2005. New mechanism contributing to druginduced arrhythmia: rescue of a misprocessed LQT3 mutant. Circulation. 112:3239-3246.

24. Isom, L.L., et al. 1995. Functional co-expression of the $b 1$ and type IIA a subunits of sodium channels in a mammalian cell line. J. Biol. Chem. 270:3306-3312.

25. Nagatomo, T., January, C.T., and Makielski, J.C. 2000. Preferential block of late sodium current in the LQT3 Delta KPQ mutant by the class IC antiarrhythmic flecainide. Mol. Pharmacol. 57:101-107.

26. Sasaki, K., et al. 2004. Unexpected mexiletine responses of a mutant cardiac $\mathrm{Na}+$ channel implicate the selectivity filter as a structural determinant of antiarrhythmic drug access. Mol. Pharmacol. 66:330-336.

27. Bean, B., Cohen, C., and Tsien, R. 1983. Lidocaine block of cardiac sodium channels. J. Gen. Physiol. 81:613-642.

28. Veldkamp, M.W., et al. 2003. Contribution of sodium channel mutations to bradycardia and sinus node dysfunction in LQT3 families. Circ. Res. 92:976-983.
29. Wang, D.W., et al. 2007. Cardiac sodium channel dysfunction in sudden infant death syndrome. Circulation. 115:368-376.

30. Deschenes, I., et al. 2000. Electrophysiological characterization of SCN5A mutations causing long QT (E1784K) and Brugada (R1512W and R1432G) syndromes. Cardiovasc. Res. 46:55-65.

31. Splawski, I., et al. 2000. Spectrum of mutations in long-QT syndrome genes. KVLQT1, HERG, SCN5A, KCNE1, and KCNE2. Circulation. 102:1178-1185

32. Tester, D.J., Will, M.L., Haglund, C.M., and Ackerman, M.J. 2005. Compendium of cardiac channel mutations in 541 consecutive unrelated patients referred for long QT syndrome genetic testing. Heart Rhythm. 2:507-517.

33. Napolitano, C., et al. 2005. Genetic testing in the long QT syndrome: Development and validation of an efficient approach to genotyping in clinical practice. JAMA. 294:2975-2980.

34. Kambouris, N.G., et al. 2000. A revised view of cardiac sodium channel "blockade" in the long-QT syndrome. J. Clin. Invest. 105:1133-1140.

35. Tellez, J.O., et al. 2006. Differential expression of ion channel transcripts in atrial muscle and sinoatrial node in rabbit. Circ. Res. 99:1384-1393.

36. Maier, S.K.G., et al. 2003. An unexpected requirement for brain-type sodium channels for control of heart rate in the mouse sinoatrial node. Proc. Natl. Acad. Sci. U. S. A. 100:3507-3512.

37. Miyazaki, T., et al. 1996. Autonomic and antiarrhythmic drug modulation of ST segment elevation in patients with Brugada syndrome. J. Am. Coll. Cardiol. 27:1061-1070.

38. Brugada, R., et al. 2000. Sodium channel blockers identify risk for sudden death in patients with STsegment elevation and right bundle branch block but structurally normal hearts. Circulation. 101:510-515.

39. Olgin, J.E., and Zipes, D.P. 2001. Specific arrhythmias: diagnosis and treatment. In Heart disease: a textbook of cardiovascular medicine. E. Braunwald, D.P. Zipes, and P. Libby, editors. W.B. Saunders. Philadelphia, Pennsylvania, USA. 815-899.

40. Wang, Q., Li, Z., Shen, J., and Keating, M.T. 1996. Genomic organization of the human SCN5A gene encoding the cardiac sodium channel. Genomics. 34:9-16.

41. Makita, N., et al. 2002. Drug-induced long-QT syndrome associated with a subclinical SCN5A mutation. Circulation. 106:1269-1274. 